\title{
Sistema Fuzzy Como Mecanismo Auxiliador na Predição da Doença Viral COVID-19
}

\author{
Roney Nogueira de Sousa ${ }^{1}$, Janaide Nogueira de Sousa Ximenes, Rhyan Ximenes de Brito ${ }^{2}$ \\ ${ }^{1}$ Instituto Federal de Educação, Ciência e Tecnologia do Ceará (IFCE) \\ CE-187, s/n - Estádio, - CEP 62320-000 - Tianguá - CE - Brasil \\ ${ }^{2}$ Faculdade IEducare (FIED) - Rua Conselheiro João Lourenço, \\ 406 - CEP 62320-000 - Tianguá - CE - Brasil \\ \{nogueiraroney453, nogueirajanaide, rxbrito\}egmail.com
}

\begin{abstract}
The fuzzy logic is suitable for the manipulation of subjective information such as that of the viral disease COVID-19, considered one of the most feared by the population due to its high rate of contamination. This work presents a system based on fuzzy rules for a pre-diagnosis, aiming to assist health professionals. The methodology was based on bibliographic research and simulations with fictitious patients, with the characteristic symptoms of the disease addressed as an assessment measure. The results were promising as they were very close to those observed by health professionals. The study showed the importance of using fuzzy logic in the pre-diagnosis of viral diseases such as COVID-19.
\end{abstract}

Resumo. A lógica fuzzy é adequados para a manipulação de informações subjetivas como as da doença viral COVID-19, considerada uma das mais temidas pela população por seu alto índice de contaminação. Este trabalho apresenta um sistema baseado em regras fuzzy para um pré-diagnóstico, objetivando auxiliar profissionais da saúde. A metodologia baseou-se em pesquisas bibliográficas e em simulações com pacientes fictícios, tendo como medida de avaliação os sintomas característicos da doença abordada. Os resultados mostraram-se promissores na medida que foram bem próximos dos observados por profissionais da saúde. O estudo mostrou a importância da utilização da lógica fuzzy no pré-diagnóstico de doenças virais como a COVID-19.

\section{Introdução}

Um surto de pneumonia de causa desconhecida foi relatado pelas autoridades de saúde na cidade de Wuhan, na China, em dezembro de 2019, que logo em seguida foi batizado de COVID-19, considerada uma doença aguda resolvível, mas que, também, pode ser mortal, principalmente para grupos de risco, como idosos e pessoas diagnosticadas com doenças crônicas, como diabetes, hipertensão, tuberculose entre outras [Campos and da Costa 2020].

A utilização dos modelos baseados em lógica clássica, mostraram-se incapazes de solucionar vários problemas e o uso da lógica difusa como extensão da lógica clássica tem possibilitado enormes avanços no tratamento de dados complexos [Goyal and Singhai 2018]. Nessa perspectiva o estudo em questão objetivou a 
implementação e análise de dados obtidos através de um sistema baseado em Lógica Fuzzy visando usá-lo como auxílio no diagnóstico da COVID-19.

A motivação para o presente estudo está relacionada a necessidade de aprimoramento de técnicas para a diagnose efetiva e precoce da COVID-19, de forma que se possa tomar medidas efetivas para o controle e combate a proliferação do vírus.

Este trabalho está dividido em sete seções: Seção 2 descreve alguns trabalhos relacionados, Seção 3 apresenta um referencial teórico com conceitos sobre a Lógica Fuzzy. A Seção 4 abordará sobre a família dos coronavírus, na Seção 5 será abordada de forma breve a doença COVID-19, na Seção 6 será descrita a abordagem proposta no trabalho, na Seção 7, apresentar-se-á os resultados e discussões baseados na abordagem proposta no trabalho e por fim, na Seção 8 é descrita a conclusão e trabalhos futuros.

\section{Trabalhos Relacionados}

Esta seção apresenta uma revisão bibliográfica de trabalhos que abordam a utilização da lógica fuzzy como ferramenta de predição para os mais variados tipos de doenças.

[Dames et al. 2004] analisou a utilização da entropia aproximada Fuzzy do padrão de vazão respiratória no diagnóstico de alterações em fase inicial na doença pulmonar obstrutiva crônica. A entropia aproximada do padrão de vazão respiratória foi medida em um grupo de 40 indivíduos, incluindo voluntários normais, fumantes e pacientes com doença pulmonar obstrutiva crônica com níveis leves de obstrução das vias aéreas. Os efeitos adversos da obstrução leve foram detectados com uma exatidão de aproximadamente $80 \%$.

[da Silva et al. 2017] construíram um sistema baseado em regras fuzzy para auxiliar na análise dos riscos de uma mulher desenvolver ou já ser portadora do câncer de mama, para construção do modelo foram consideradas três variáveis: a idade da paciente, os fatores de risco e os sintomas (se houver). A variável idade foi classificada como: muito jovem, jovem, madura e avançada. A variável idade mostrou total relevância pois, quanto maior a idade, maiores são as chances de desenvolvimento da doença.

[Almeida Araujo and Ximenes de Brito 2020] apresentaram um sistema baseado em regras fuzzy objetivando auxiliar psiquiatras no diagnóstico da doença. A metodologia baseou-se em pesquisas bibliográficas e em simulações com pacientes fictícios, foram utilizadas como variáveis de entrada sintomas como delírios, alucinações, discurso desorganizado, entre outras. Os resultados mostraram-se promissores na medida que foram bem próximos dos observados por profissionais da saúde.

[Ximenes et al. 2019] teve por objetivo realizar um estudo sobre a meningite e assim modelar um sistema Fuzzy para auxiliar no diagnóstico da doença, tendo como medida de avaliação os sintomas característicos da doença. Foram utilizadas como variáveis linguísticas, febre, calafrios, fotofobia, náusea, vômitos, status mental alterado e rigidez no pescoço, utilizou-se além dos sintomas, o método de inferência Sugeno, com níveis de gravidade para os sintomas, alto, moderado, baixo e baixíssimo.

\section{Lógica Fuzzy}

A lógica nebulosa é baseada na teoria dos conjuntos Fuzzy. Ela difere dos sistemas lógicos tradicionais em suas características e seus detalhes. Nesta lógica, o raciocínio exato cor- 
responde a um caso limite do raciocínio aproximado, sendo interpretado como um processo de composição de relações nebulosas [Sandri et al. 1999].

Segundo [Júnior and Novakowski 2005] os conjuntos Fuzzy foram desenvolvidos para determinar o quanto um elemento pertence ou não a um determinado conjunto, com o uso de graus de pertinência, que são valores no intervalo [0;1]. Assim é possível determinar os elementos que pertencem a um grupo e seu respectivo grau de pertinência.

Na lógica Fuzzy pode ser observado que o valor verdade de uma proposição pode ser um subconjunto Fuzzy de qualquer conjunto parcialmente ordenado, ao contrário dos sistemas lógicos binários, onde o valor verdade só pode assumir dois valores: verdadeiro (1) ou falso (0). Nos sistemas difusos o valor verdade de uma proposição pode ser ou um elemento de um conjunto finito, num intervalo, ou uma álgebra booleana [Gomide and Gudwin 1994].

\section{Coronavírus}

Os COVs podem ser encontrados em vários hospedeiros, mas são os morcegos os principais reservatórios desses vírus. Outros animais como aves, mamíferos, incluindo camelos, civetas de palma (gatos selvagens asiáticos), ratos, cães, gatos, cobras também podem ser reservatórios dos COVs [Chaves and Bellei 2020].

Os primeiros casos de coronavírus em humanos foram identificados e isolados em 1937, porém somente em 1965 o vírus foi denominado por coronavírus, devido ao seu perfil microscópico parecer com uma coroa [Cabral et al. 2020]. Nos últimos vinte anos o coronavírus foi responsável por duas pandemias importantes, a Severe Acute Respiratory Syndrome (SARS)-CoV, em 2002 e a Middle East Respiratory Syndrome (MERS)-CoV em 2012 [de Campos Tuñas et al. 2020].

Os Coronavírus são causadores de infecções respiratórias em uma variedade de animais, incluindo aves e mamíferos. Sete coronavírus são reconhecidos como patógenos em humanos [Lana et al. 2020]. Como vírus RNA, representam a maioria dos vírus presentes na natureza, no mundo vegetal, animal e na espécie humana [Chaves and Bellei 2020].

\section{1. $S A R S-C o V-2$}

Em dezembro de 2019, um grupo de pacientes com pneumonia de causa desconhecida foi vinculado a um mercado atacadista de frutos do mar em Wuhan, China [Zhu et al. 2020], os resultados da investigação laboratorial das amostras, identificaram um novo coronavírus como responsável pelo surto, conhecido provisoriamente como 2019-nCoV [Chaves and Bellei 2020], em 11 de fevereiro recebeu o nome de SARS-CoV2 [Arrais et al. 2020].

Salienta-se que o vírus SARS-CoV-2 possui estrutura membranosa de espinhos proteicos e penetra nas células através dos receptores celulares da enzima conversora de angiotensina 2 [de Campos Tuñas et al. 2020].

\section{A Doença COVID-19}

A doença que o novo coronavírus produz é a COVID-19, onde 'CO' significa corona, 'VI' para vírus e 'D' para doença. Antigamente, essa doença era chamada de "2019 novo Coronavírus" ou "2019-nCoV" [de Campos Tuñas et al. 2020]. 
Os sintomas iniciais lembram uma gripe, no entanto, de indivíduo para indivíduo, a sintomatologia é muito variada, desde pessoas assintomáticas ou com sintomas leves até manifestações mais graves que levam à morte, especialmente em idosos com comorbidades, ou seja, doença preexistentes [Dolce Filho et al. 2020].

De acordo com a Organização Mundial da Saúde (OMS) os sintomas podem ser febre, tosse seca, cansaço, dor de garganta, diarréia, conjuntivite, dor de cabeça, perda de paladar ou olfato, erupção cutânea ou descoloração dos dedos das mãos ou dos pés, dificuldade em respirar ou falta de ar, dor ou pressão no peito, entre outros sintomas [da Saúde 2020].

Com relação a transmissão observa-se um alto índice de contágio ocorrendo através de contato próximo, seja por meio de gotículas de saliva ou por toque em superfícies contaminadas [Franco et al. 2020] [Cabral et al. 2020] com transmissibilidade em média de 7 a 14 dias após o início dos sintomas [Baêta et al. 2020].

\section{Abordagem Proposta}

Com o auxílio do software MatLab foi modelado um sistema com base em lógica Fuzzy para a realização das simulações, onde foram utilizados como dados de entrada sintomas de pacientes fictícios. A Figura 1 mostra a estrutura do sistema Fuzzy modelado, com variáveis de entrada, as regras de fuzzyficação e as variáveis de saída.

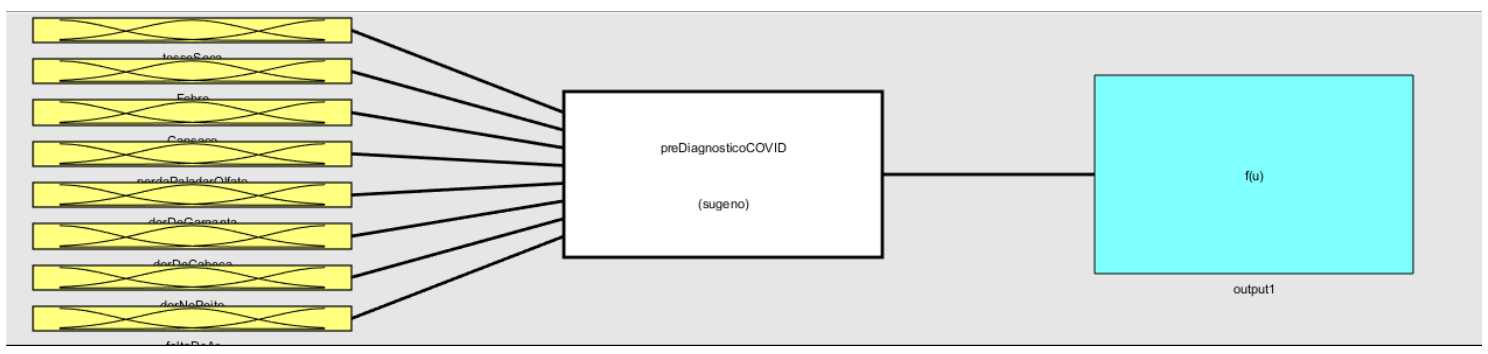

Figura 1. Variáveis de Entrada

A Figura 2 demonstra a definição das regras as quais foram necessárias para obter o diagnóstico da COVID-19. Foram elaboradas 820 regras de inferência para obter todos os possíveis resultados de combinações entre as entradas e as saídas esperadas. A partir destas regras foi possível identificar se o paciente analisado tem ou não o risco de ter a doença e o nível de gravidade que se apresenta.

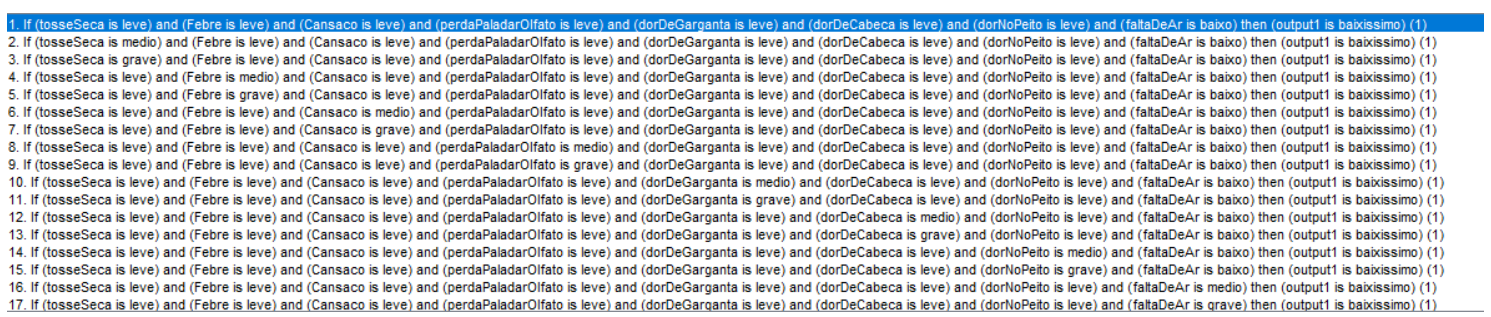

Figura 2. Regras de Inferência do Sistema Fuzzy

Durante a análise foram selecionados os seguintes sintomas: tosse, febre, cansaço, perda de olfato e paladar, dor de garganta, dor de cabeça, dor no peito, falta de ar. Estes sintomas foram considerados como variáveis de entrada do sistema Fuzzy, o método 
de inferência utilizado foi o Método de Sugeno. Os valores linguísticos utilizados nas variáveis de entrada foram: leve, médio e grave.

\section{Resultados e Discussões}

A Tabela 1 mostra os sintomas com os respectíveis níveis de gravidades de um paciente $\mathrm{X}$, a qual foi verificado que alguns sintomas apresentaram-se na fase leve, tal como: febre apresentando 0.37, perda de olfato e paladar com 0.23. Porém outros sintomas apresentaram-se à fase moderada como: tosse seca 0.39 , dor de garganta 0.61 . Também foi observado para o sintoma dor no peito 0.67 na fase mediana com aproximação grave. Por fim foram observados os seguintes sintomas na fase grave: cansaço 0.81, dor de cabeça 0.74 e falta de ar com 0.79 .

Tabela 1. Valores Para Pré-diagnóstico Paciente $X$

\begin{tabular}{|l|c|}
\hline Sintomas & Gravidade \\
\hline Tosse seca & 0.39 \\
\hline Febre & 0.37 \\
\hline Cansaço & 0.81 \\
\hline Perda de olfato e paladar & 0.23 \\
\hline Dor de garganta & 0.61 \\
\hline Dor de cabeça & 0.74 \\
\hline Dor no peito & 0.67 \\
\hline Falta de ar & 0.79 \\
\hline
\end{tabular}

Na Figura 3 são apresentados os resultados obtidos com a simulação de diagnóstico da COVID-19, obtido através dos resultados de todos os sintomas aplicando as regras de inferência do sistema Fuzzy. Assim o resultado obtido foi de 0.75 demonstrando que o paciente $\mathrm{X}$ se encontra com alto risco de ter a doença. Dessa forma devendo ser submetido a exames laboratórias para melhor dimensionamento dos riscos e confirmação da suspeita de modo a ser encaminhado para o tratamento mais adequando.

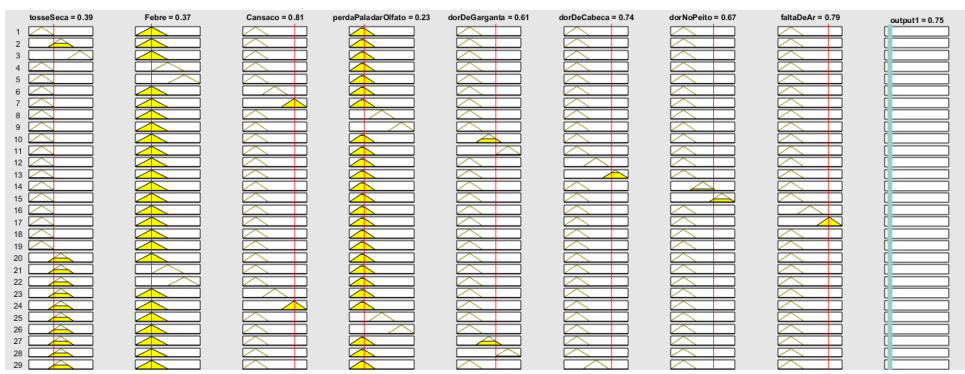

Figura 3. Pré-diagnóstico Paciente $X$

A Tabela 2 mostra os sintomas e os níveis de gravidade de um paciente Y, verificando para o sintoma da febre 0.36, fase leve. Porém outros sintomas apresentaram-se na fase leve com aproximação mediana como perda de olfato e paladar, dor de cabeça e dor no peito, todos com 0.33 e por fim a falta de ar 0.31 . Também foi observado sintomas na fase mediana como, tosse seca 0.42 e dor de garganta 0.51 . Por fim foi observado o sintoma cansaço 0.65 , na fase mediana com aproximação grave. 
Tabela 2. Valores Para Pré-diagnóstico Paciente Y

\begin{tabular}{|l|c|}
\hline Sintomas & Gravidade \\
\hline Tosse seca & 0.42 \\
\hline Febre & 0.365 \\
\hline Cansaço & 0.65 \\
\hline Perda de olfato e paladar & 0.33 \\
\hline Dor de garganta & 0.51 \\
\hline Dor de cabeça & 0.33 \\
\hline Dor no peito & 0.33 \\
\hline Falta de ar & 0.31 \\
\hline
\end{tabular}

Na Figura 4 são apresentados os resultados obtidos com a realização da simulação de diagnóstico da COVID-19, obtido através dos resultados de todos os sintomas aplicando as regras de inferência do sistema Fuzzy. Assim o resultado obtido foi de 0.29 demonstrando que o paciente Y se encontra com baixo risco de ter a doença. Porém ficando a cargo do profissional de saúde orientar o paciente para possíveis medidas de isolamento social ou quarentena.

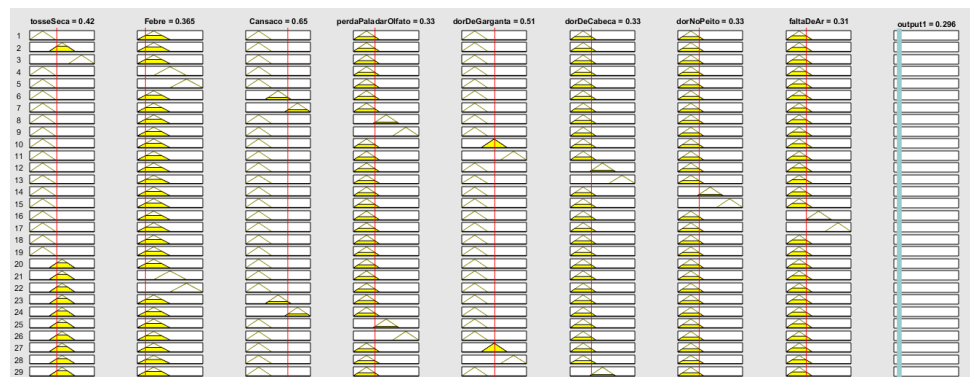

Figura 4. Pré-diagnóstico Paciente $Y$

A Tabela 3 mostra os sintomas e os níveis de gravidades de um paciente Z também fictício, constatando sintomas na fase leve como, perda de paladar e olfato 0.29. Outros sintomas na fase leve com aproximação para mediana como falta de ar com 0.32. Observou-se também sintomas na fase mediana como, tosse seca 0.51 , cansaço 0.54 , dor de garganta 0.56 e por fim dor no peito com 0.44 . Foram observados os seguintes sintomas na fase mediana com aproximação grave, febre com 0.38 . Por fim dor de cabeça com 0.72 , na fase grave.

Na Figura 5 são apresentados os resultados obtidos com a realização de uma simulação de diagnóstico da COVID-19, obtido através dos resultados de todos os sintomas aplicando as regras de inferência do sistema Fuzzy. Assim o resultado obtido foi de 0.29 demonstrando que o paciente $\mathrm{Z}$ se encontra com baixo risco de ter a doença. Com esse pré-diagnóstico um profissional de saúde terá dados para conversar confortavelmente com o paciente orientando-o sobre as medidas que garantem o combate a doença.

\section{Conclusão e Trabalhos Futuros}

O estudo em questão foi desenvolvido com o intuito de auxiliar no processo de prédiagnóstico da COVID-19, embora as simulações tenham sido realizadas com pacientes 
Tabela 3. Valores Para Pré-diagnóstico Paciente Z

\begin{tabular}{|l|c|}
\hline Sintomas & Gravidade \\
\hline Tosse seca & 0.42 \\
\hline Febre & 0.365 \\
\hline Cansaço & 0.65 \\
\hline Perda de olfato e paladar & 0.33 \\
\hline Dor de garganta & 0.51 \\
\hline Dor de cabeça & 0.33 \\
\hline Dor no peito & 0.33 \\
\hline Falta de ar & 0.31 \\
\hline
\end{tabular}

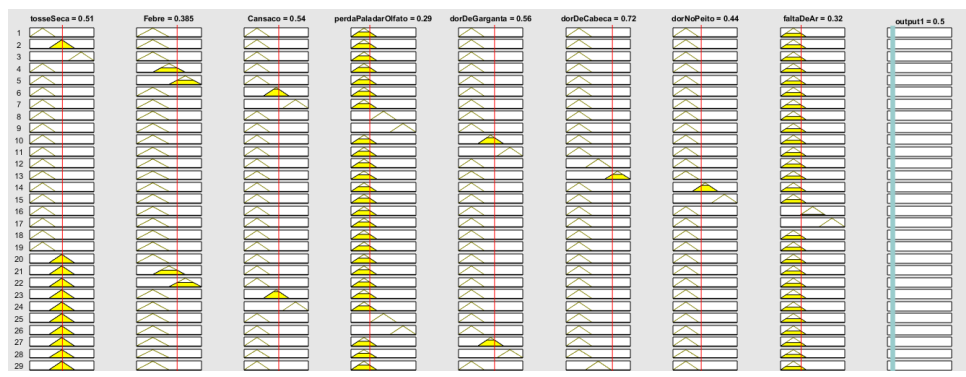

Figura 5. Pré-diagnóstico Paciente Z

fictícios, o sistema pode ser validado com dados reais e adaptado para produzir resultados confiáveis que poderão ser utilizados por profissionais da saúde para auxílio na detecção precocemente.

Para trabalhos futuros sugere-se o desenvolvimento de outro sistema Fuzzy, utilizando o método Mandani, visando a comparação dos resultados dos testes e logo após utilizar redes neurais afim de obter o melhor resultado para a problemática em questão.

\section{Referências}

Almeida Araujo, M. D. and Ximenes de Brito, R. (2020). Modelo de previsão fuzzy como mecanismo de pré-diagnóstico da esquizofrenia em adultos. Revista de Sistemas e Computação-RSC, 10(1).

Arrais, T. P. A., Oliveira, A. R. d., Alencar, D. P., Salgado, T. R., Lima, L. d. O., Viana, J. L. R., Miranda, A. F. d., Campos, F. R., Arriel, M. F., Borges, L. J., et al. (2020). Pandemia covid-19: o caráter emergencial das transferências de renda direta e indireta para a população vulnerável do estado de goiás.

Baêta, K. F., Suassuna, T. d. S., Buralli, R. J., Almeida, W. A. F. d., Costa, M. M. d. M., Oliveira, L. S. d. C. d., Tavares, G., Wollmann, L., Júnior, A., Cezáreo, A., et al. (2020). Protocolo de tratamento do novo coronavírus (2019-ncov).

Cabral, R. B. G., Chase, S. A. N., Ribeiro, R. C. M., Marques, G. T., Morais, E. C., de Jesus Zissou, A., Andrade, E. d. S. S., Couto, W. O., dos Santos Júnior, P. C., da Silva Campos, P. S., et al. (2020). Estudo inicial sobre a evolução do novo coronavírus (sars-cov-2) no estado do pará (brasil), no período entre 17/03/2020 e $06 / 04 / 2020 /$ initial study on the evolution of the new coronavirus (sars-cov-2) in the 
state of pará (brazil), in the period between 03/17/2020 and 06/04/2020. Brazilian Journal of Health Review, 3(2):2914-2931.

Campos, N. G. and da Costa, R. F. (2020). Alterações pulmonares causadas pelo novo coronavírus (covid-19) e o uso da ventilação mecânica invasiva. Journal of Health \& Biological Sciences, 8(1):1-3.

Chaves, T. d. S. S. and Bellei, N. C. J. (2020). Sars-cov-2, o novo coronavírus. Revista de Medicina, 99(1):i-iv.

da Saúde, O. M. (2020). Coronavirus. disponivel em: https://www.who.int/healthtopics/coronavirus\#tab=tab_3. acessado em 02/05/2020.

da Silva, P., de Brito, R., de Sousa Ximenes, J., and de Sousa, R. (2017). A aplicaçao da lógica de fuzzy no auxılio do diagnóstico do câncer de mama.

Dames, K., Mattos, R., and Melo, P. (2004). Aprimoramento da entropia fuzzy da vazão respiratória visando o diagnóstico precoce da doença pulmonar obstrutiva crônica.

de Campos Tuñas, I. T., da Silva, E. T., Santiago, S. B. S., Maia, K. D., and Silva-Júnior, G. O. (2020). Doença pelo coronavírus 2019 (covid-19): Uma abordagem preventiva para odontologia. Revista Brasileira de Odontologia, 77:1-7.

Dolce Filho, R., Nechar, R. C., and Ribeiro Filho, A. (2020). Estudo preliminar de sintomas e medicamentos prevalentes do gênio epidêmico da pandemia de covid-19 no brasil.

Franco, A. G., Franco, A. B. G., de Carvalho, G. A. P., Ramos, E. V., and Dias, S. C. (2020). Máscaras cirúrgicas em tempos de coronavírus. InterAmerican Journal of Medicine and Health, 3:e202003003-e202003003.

Gomide, F. A. C. and Gudwin, R. R. (1994). Modelagem, controle, sistemas e lógica fuzzy. SBA controle \& Automação, 4(3):97-115.

Goyal, K. and Singhai, J. (2018). Review of background subtraction methods using gaussian mixture model for video surveillance systems. Artificial Intelligence Review, 50(2):241-259.

Júnior, I. and Novakowski, S. (2005). Lógica fuzzy sistemas de apoio a decisão. Rio Grande do Sul, UNISINOS, Abr.

Lana, R. M., Coelho, F. C., Gomes, M. F. d. C., Cruz, O. G., Bastos, L. S., Villela, D. A. M., and Codeço, C. T. (2020). Emergência do novo coronavírus (sars-cov-2) e o papel de uma vigilância nacional em saúde oportuna e efetiva. Cadernos de Saúde Pública, 36:e00019620.

Sandri, S., Correa, C., and Nebulosa, L. (1999). V escola de redes neurais. ITA (Instituto Tecnológico da Aeronáutica), pp. c073-c090.

Ximenes, J., de Sousa, R., and de Brito, R. (2019). Sistema fuzzy como instrumento de auxílio no pré-diagnóstico da meningite. In Anais da VII Escola Regional de Computação Aplicada à Saúde, pages 264-269, Porto Alegre, RS, Brasil. SBC.

Zhu, N., Zhang, D., Wang, W., Li, X., Yang, B., Song, J., Zhao, X., Huang, B., Shi, W., Lu, R., et al. (2020). A novel coronavirus from patients with pneumonia in china, 2019. New England Journal of Medicine. 